Georgian Mathematical Journal

Volume 13 (2006), Number 4, 737-740

\title{
ON THE EISENSTEIN SERIES CORRESPONDING TO QUADRATIC FORMS OF CERTAIN TYPE
}

\author{
NIKOLOZ KACHAKHIDZE
}

\begin{abstract}
The Eisenstein series corresponding to quadratic forms of type $(f / 2,4 N, \chi)(N$ is a square-free natural number) are constructed using the bases of the spaces of Eisenstein series given in [4].
\end{abstract}

2000 Mathematics Subject Classification: 11F11, 11E20.

Key words and phrases: Eisenstein series, quadratic forms.

We will use the notation and notions from [3] and [4].

In [5] Malyshev constructed a Hardy-Littlewood singular series for any integral positive quadratic form with $f \geq 4$ variables. In [2] Beridze summed up this series. But the use of this result to obtain Fourier coefficients of this series for a given quadratic form needs very long and tedious calculations.

As is known (see [7], p. 160), for any entire modular form $F(\tau)$ there exists a linear combination of Eisenstein series $E(\tau)$ such that $F(\tau)-E(\tau)$ is a cusp form. In this paper, using the results of [4], we find a linear combination of Eisenstein series $E(\tau, Q(x))$ such that $\vartheta(\tau, Q(x))-E(\tau, Q(x))$ is a cusp form of a certain type.

In what follows, let $f, M \in \mathbb{N}, N=p_{1} p_{2} \cdots p_{j}$ ( $p_{l}$ is an odd prime number, $l=1,2, \ldots, j ; p_{l_{1}} \neq p_{l_{2}}$ when $\left.l_{1} \neq l_{2}\right)$. If $Q(X)$ is a quadratic form with integral coefficients and with $f$ variables $m \in \mathbb{N}, g \in \mathbb{Z}^{f}$, then

$$
S(Q, m)=\sum_{g \bmod m} \exp \left(\frac{2 \pi i}{m} Q(g)\right) .
$$

Lemma ([7], p. 213; [6]). Let $L=\left(\begin{array}{ll}a & b \\ c & d\end{array}\right) \in S L_{2}(\mathbb{Z}), c \neq 0 ; Q(X)$ be a quadratic form of type $(f / 2, M, \chi), A$ be the matrix of $2 Q(X) ; h, g \in \mathbb{Z}^{f}$. Then

$$
\vartheta(L \tau, Q(X))=\frac{(\sqrt{-i(c \tau+d) \operatorname{sgn} c})^{f}}{\sqrt{\operatorname{det} A} \cdot|c|^{f / 2}} \sum_{\substack{h \bmod M \\ h A \equiv(\bmod M)}} \varphi_{h}^{L} \vartheta(\tau ; Q(X), 1, h),
$$

where

$$
\varphi_{h}^{L}=\sum_{g \bmod |c|} \exp \left(\frac{2 \pi i}{c}\left(a Q(g)+\frac{1}{M} \operatorname{sgn} c \cdot g A h^{\prime}+\frac{d}{M^{2}} Q(h)\right)\right) .
$$

Proposition 1. Let $2 \nmid f, f \geq 5, Q(X)$ be a quadratic form of type $(f / 2,4 N, \chi)$ and 


$$
\begin{aligned}
& E(\tau, Q(X))=E_{1}(\tau ; f / 2,4 N, \chi)+\frac{\exp (\pi i f / 4)}{\sqrt{\operatorname{det} A}}\left(\sum_{\substack{N_{l} \mid N \\
N_{l} \neq N}} 2^{-f} N_{l}^{-f / 2} \overline{S\left(Q, 4 N_{l}\right)}\right. \\
& \left.\quad \times E_{2, l}(\tau ; f / 2,4 N, \chi)+\sum_{N_{l} \mid N}\left(\frac{-1}{N_{l}}\right) N_{l}^{-f / 2} \overline{S\left(Q, N_{l}\right)} E_{3, l}(\tau ; f / 2,4 N, \chi)\right),
\end{aligned}
$$

where $E_{1}(\tau ; f / 2,4 N, \chi)$ and $E_{r, l}(\tau ; f / 2,4 N, \chi)(r=2,3)$ are defined in [4]. Then

$$
\vartheta(\tau, Q(X))-E(\tau, Q(X)) \in S_{f / 2}\left(\widetilde{\Gamma}_{0}(4 N), \chi\right) .
$$

Proof. It follows from Proposition 2 of [4] and Theorem 2.2, p. 86, of [1] that

$$
\vartheta(\tau, Q(X))-E(\tau, Q(X)) \in M_{f / 2}\left(\widetilde{\Gamma}_{0}(4 N), \chi\right) .
$$

Therefore we must only show that $\vartheta(\tau, Q(X))$ and $E(\tau, Q(X))$ assume equal values at cusps. The group $\Gamma_{0}(4 N)$ has $2^{j+1} f$-regular cusps (see [4]). Let $\widetilde{L}_{1}=$ $\left(\left(\begin{array}{ll}1 & 0 \\ 0 & 1\end{array}\right), 1\right), \widetilde{L}_{2, l}=\left(\left(\begin{array}{cc}1 & 0 \\ -4 N_{l} & 1\end{array}\right), \sqrt{-4 N_{l} \tau+1}\right)\left(N_{l} \mid N, N_{l} \neq N\right), \widetilde{L}_{3, l}=$ $\left(\left(\begin{array}{cc}1 & 0 \\ -N_{l} & 1\end{array}\right),-i \varepsilon_{N_{l}} \sqrt{-N_{l} \tau+1}\right)\left(N_{l} \mid N\right) ; A$ be the matrix of $2 Q(X), h \in \mathbb{Z}^{f}$. Using the Lemma we get

$$
\begin{aligned}
& \lim _{\tau \rightarrow i \infty}\left(\left.\vartheta(\tau, Q(X))\right|_{f / 2} \widetilde{L}_{1}\right)=1, \\
& \lim _{\tau \rightarrow i \infty}\left(\left.\vartheta(\tau, Q(X))\right|_{f / 2} \widetilde{L}_{2, l}\right)=\lim _{\tau \rightarrow i \infty}\left(\frac{1}{\sqrt{\operatorname{det} A}\left(4 N_{l}\right)^{f / 2}} \cdot\left(\frac{\sqrt{i\left(-4 N_{l} \tau+1\right)}}{\sqrt{-4 N_{l} \tau+1}}\right)^{f}\right. \\
& \left.\times \sum_{\substack{h \bmod 4 N \\
h A \equiv(\bmod 4 N)}} \varphi_{h}^{L_{2, l}} \vartheta(\tau ; Q(X), 1, h)\right) \\
& =\frac{\exp (\pi i f / 4)}{\sqrt{\operatorname{det} A}\left(4 N_{l}\right)^{f / 2}} \sum_{g \bmod 4 N_{l}} \exp \left(-\frac{2 \pi i}{4 N_{l}} Q(g)\right) \\
& =\frac{\exp (\pi i f / 4)}{\sqrt{\operatorname{det} A}\left(4 N_{l}\right)^{f / 2}} \overline{S\left(Q, 4 N_{l}\right)}, \\
& \lim _{\tau \rightarrow i \infty}\left(\left.\vartheta(\tau, Q(X))\right|_{f / 2} \widetilde{L}_{3, l}\right)=\lim _{\tau \rightarrow i \infty}\left(\frac{1}{\sqrt{\operatorname{det} A} N_{l}^{f / 2}} \cdot\left(\frac{\sqrt{i\left(-N_{l} \tau+1\right)}}{-i \varepsilon_{N_{l}} \sqrt{-N_{l} \tau+1}}\right)^{f}\right. \\
& \left.\times \sum_{\substack{h \bmod 4 N \\
h A=0(\bmod 4 N)}} \varphi_{h}^{L_{3, l}} \vartheta(\tau ; Q(X), 1, h)\right) \\
& =\frac{i^{f} \exp (\pi i f / 4)}{\sqrt{\operatorname{det} A} N_{l}^{f / 2} \varepsilon_{N_{l}}^{f}} \sum_{g} \exp \left(-\frac{2 \pi i}{N_{l}} Q(g)\right)
\end{aligned}
$$




$$
=\frac{i^{f} \exp (\pi i f / 4)}{\sqrt{\operatorname{det} A} N_{l}^{f / 2} \varepsilon_{N_{l}}^{f}} \overline{S\left(Q, N_{l}\right)} .
$$

It can be readily seen that

$$
\begin{aligned}
& \lim _{\tau \rightarrow i \infty}\left(\left.E_{1}(\tau ; f / 2,4 N, \chi)\right|_{f / 2} \widetilde{L}_{1}\right)=1, \\
& \lim _{\tau \rightarrow i \infty}\left(\left.E_{1}(\tau ; f / 2,4 N, \chi)\right|_{f / 2} \widetilde{L}_{r, l}\right)=0, \\
& \lim _{\tau \rightarrow i \infty}\left(\left.E_{r, l}(\tau ; f / 2,4 N, \chi)\right|_{f / 2} \widetilde{L}_{1}\right)=0 \quad(r=2,3), \\
& \lim _{\tau \rightarrow i \infty}\left(\left.E_{2, l}(\tau ; f / 2,4 N, \chi)\right|_{f / 2} \widetilde{L}_{2, l}\right)=1, \\
& \lim _{\tau \rightarrow i \infty}\left(\left.E_{3, l}(\tau ; f / 2,4 N, \chi)\right|_{f / 2} \widetilde{L}_{3, l}\right)=i^{f}\left(\frac{-1}{N_{l}}\right), \\
& \lim _{\tau \rightarrow i \infty}\left(\left.E_{r_{1}, l_{1}}(\tau ; f / 2,4 N, \chi)\right|_{f / 2} \widetilde{L}_{r_{2}, l_{2}}\right)=0 \text { when } r_{1}, r_{2}=2 \\
& \text { or } 3 \text { and }\left(r_{1}, l_{1}\right) \neq\left(r_{2}, l_{2}\right) .
\end{aligned}
$$

Then the result follows from (1)-(10).

Proposition 2. Let $Q(X)$ be a quadratic form of type $(f / 2,4 N, \chi), 2 \mid f$, $\chi=\phi \psi, \phi$ be a character $\bmod 4, \psi$ be a character $\bmod N$,

$$
\begin{aligned}
E(\tau, Q(X)) & =E_{1}(\tau ; f / 2,4 N, \chi)+\frac{i^{f / 2}}{\sqrt{\operatorname{det} A}}\left(\sum_{\substack{N_{l} \mid N \\
N_{l} \neq N}} 2^{-f} N_{l}^{-f / 2} \overline{S\left(Q, 4 N_{l}\right)}\right. \\
& \left.\times E_{2, l}(\tau ; f / 2,4 N, \chi)+\sum_{N_{l} \mid N} N_{l}^{-f / 2} \overline{S\left(Q, N_{l}\right)} E_{3, l}(\tau ; f / 2,4 N, \chi)\right)
\end{aligned}
$$

if $\phi$ is not the principal character $\bmod 4$ and

$$
\begin{aligned}
& E(\tau, Q(X))=E_{1}(\tau ; f / 2,4 N, \chi)+\frac{i^{f / 2}}{\sqrt{\operatorname{det} A}}\left(\sum_{\substack{N_{l} \mid N \\
N_{l} \neq N}} 2^{-f} N_{l}^{-f / 2} \overline{S\left(Q, 4 N_{l}\right)}\right. \\
& \times E_{2, l}(\tau ; f / 2,4 N, \chi)+\sum_{N_{l} \mid N}\left(N_{l}^{-f / 2} \overline{S\left(Q, N_{l}\right)} E_{3, l}(\tau ; f / 2,4 N, \chi)\right. \\
& \left.\left.+\left(2 N_{l}\right)^{-f / 2} \overline{S\left(Q, 2 N_{l}\right)} E_{4, l}(\tau ; f / 2,4 N, \chi)\right)\right)
\end{aligned}
$$

if $\phi$ is the principal character mod 4, where

$$
E_{1}(\tau ; f / 2,4 N, \chi) \quad \text { and } \quad E_{r, l}(\tau ; f / 2,4 N, \chi) \quad(r=2,3,4)
$$

are defined in [4]. Then

$$
\vartheta(\tau, Q(X))-E(\tau, Q(X)) \in S_{f / 2}\left(\widetilde{\Gamma}_{0}(4 N), \chi\right) .
$$

This Proposition is proved in the same manner as Proposition 1. 


\section{REFERENCES}

1. A. N. Andrianov and V. G. Zuravlev, Modular forms and Hecke operators. (Russian) Nauka, Moscow, 1990.

2. R. I. BERIDzE, On the summation of the singular series of Hardy-Littlewood. (Russian) Soobshch. Akad. Nauk Gruzin. SSR 38(1965), 529-534.

3. E. Hecke, Mathematische Werke. Vandenhoeck \& Ruprecht, Göttingen, 1970.

4. N. KaChaKhidze, On the Fourier expansions of Eisenstein series of some types. Georgian Math. J. 13(2006), No. 1, 55-78.

5. A. Malyshev, On the representation of integers by positive quadratic forms. (Russian) Trudy Mat. Inst. Steklov 65(1962), 212 pp.

6. W. Pfetzer, Die Wirkung der Modulsubstitutionen auf mehrafache Thetareihen zu quadratischen Formen ungerader Variablenzahl. Arch. Math. 4(1953), 448-454.

7. B. Schoeneberg, Elliptic modular functions: an introduction. (Translated from the German) Die Grundlehren der mathematischen Wissenschaften, Band 203. Springer-Verlag, New York-Heidelberg, 1974.

(Received 28.07.2006)

Author's address:

Faculty of Informatics and Control Systems

Georgian Technical University

77, M. Kostava St., Tbilisi 0193

Georgia

E-mail: nika3966@yahoo.com 